\title{
Peri-procedural hemostasis disorders in surgical and transcatheter aortic valve implantation
}

\author{
Katarzyna Czerwińska-Jelonkiewicz ${ }^{1}$, Krzysztof Milewski ${ }^{1}$, Piotr Buszman ${ }^{1}$, Przemysław Kwasiborski², \\ Krzysztof Sanetra ${ }^{3}$, Wojciech Domaradzki ${ }^{3}$ Paweł Buszman ${ }^{1}$
}

${ }^{1}$ Center for Cardiovascular Research and Development, American Heart of Poland Inc., Bielsko-Biala, Poland

${ }^{2}$ Third Department of Internal Diseases and Cardiology, Warsaw Medical University, Warsaw, Poland

${ }^{3}$ First Department of Cardiac Surgery, American Heart of Poland Inc., Bielsko-Biala, Poland

Adv Interv Cardiol 2019; 15, 2 (56): 176-186

DOI: https://doi.org/10.5114/aic.2019.83649

\begin{abstract}
A bstract
Introduction: Despite their high effectiveness, surgical aortic valve replacement (AVR) and transcatheter aortic valve implantation (TAVI) are associated with substantial risk of bleeding. Although procedure-related hemostasis disorders might be crucial for safety of both procedures, the amount of data on the peri-procedural status of hemostasis in patients with aortic valve stenosis (AS) subjected to AVR and TAVI is negligible.

Aim: To investigate the profile of peri-procedural hemostasis in elderly patients with AS, subjected to aortic valve prosthesis implantation.

Material and methods: We performed a prospective analysis of global hemostasis using ROTEM thromboelastometry and platelet reactivity assessment using impedance aggregometry in 30 consecutive patients $\geq 70$ years old subjected to AVR and TAVI. All tests were performed within $24 \mathrm{~h}$ before, directly and $24 \mathrm{~h}$ after the procedures.

Results: Surgical aortic valve replacement was characterized by transient hypofibrinogenemia and von Willebrand factor (vWF) depletion, which quickly recovered within $24 \mathrm{~h}$ after AVR. Transcatheter aortic valve implantation was characterized by substantial alteration of platelet function and VWF depletion with significant platelet reactivity impairment and increase in platelet sensitivity to antiplatelet agent, early after the procedure. TAVI-related hemostasis alterations were not recovered at $24 \mathrm{~h}$ after the procedure.

Conclusions: Surgical and transcatheter aortic valve replacement procedures are associated with substantial and diverse peri-procedural hemostasis disorders. Since hemostasis disorders related to TAVI are mainly characterized by impaired platelet function, early dual antiplatelet prophylaxis after TAVI requires careful consideration.
\end{abstract}

Key words: aortic valve replacement, transcatheter aortic valve implantation, bleeding complications, peri-procedural hemostasis disorders, platelet reactivity.

Su m m a ry

Elderly patients with severe aortic valve stenosis subjected to surgical (AVR) and transcatheter aortic valve implantation (TAVI) are exposed to increased risk of bleeding. Although procedure-related hemostasis disorders might be crucial for safety of both procedures, the amount of data is negligible. We performed peri-procedural analyses of global hemostasis and platelet reactivity in 30 patients $\geq 70$ years old subjected to AVR and TAVI. The surgical procedure was characterized by quickly recovered hypofibrinogenemia and von Willebrand factor depletion. Since TAVI was mainly characterized by platelet dysfunction with increased sensitivity to antiplatelet agents, early dual antiplatelet prophylaxis after TAVI requires careful consideration.

\section{Introduction}

Severe degenerative aortic valve stenosis (AS) induces multidirectional hemostasis disorders [1-6]. The most investigated are acquired von Willebrand (vWF) syndrome with multimers depletion and enhanced platelet activation associated with high shear forces and ongoing inflammation in aortic valve tissue [1-6]. The only proven methods of AS treatment are surgical aortic valve

\section{Corresponding author:}

Katarzyna Czerwińska-Jelonkiewicz, Center for Cardiovascular Research and Development, American Heart of Poland Inc., Bielsko-Biala, Poland, phone: +48 606 858566, e-mail: kasia_czerwinska@vp.pl

Received: 2.01.2019, accepted: 13.02.2019. 
replacement (AVR) and transcatheter aortic valve implantation (TAVI) [7]. Despite the high effectiveness of these procedures, both approaches are invariably burdened with a risk of serious, procedure-related bleeding [7-11]. Procedure-related hemostasis disorders that overlap on initial coagulopathies might be crucial for the safety of both interventions. Yet, the amount of data on the peri-procedural status of hemostasis in patients subjected to AVR and TAVI is negligible [3, 12-22].

\section{Aim}

Therefore, the main aim of our study was to investigate the profile of peri-procedural hemostasis in elderly patients with AS, subjected to AVR and TAVI. Despite the obvious differences in both methods of treatment, we additionally compare hemostasis disorders associated with the procedures.

\section{Material and methods}

We carried out a prospective, single-center, nonrandomized, explorative and hypothesis-generating study with 30 patients subjected to aortic valve implantation. All consecutive patients scheduled for elective isolated TAVI or AVR between March 2017 and October 2017 were screened for eligibility. Inclusion and exclusion criteria are described in Table I. The decision about the type of aortic valve procedure was based on the current guidelines, and was reached by our heart team consisting of an interventional cardiologist and a cardiac surgeon [7].

\section{Procedures and peri-procedural anticoagulation}

All procedures were performed in the $1^{\text {st }}$ Department of Cardiac Surgery, American Heart of Poland Inc., in Bielsko-Biala, Poland, under general anesthesia. All TAVI procedures were performed with surgical access via the femoral artery, and the use of CoreValve Medtronic prostheses. All AVR were performed with median sternotomy followed by cannulation of the aorta and the right atrium access for extracorporeal circulation, using Medtronic Hancock II, Medtronic Mosaic, and St Jude Epic prostheses.

The anticoagulation protocol for AVR and TAVI was based on unfractionated heparin (UFH). During the TAVI procedure $70-100 \mathrm{IU} / \mathrm{kg}$ body weight of UFH was administered with a target activated clotting time (ACT) of 250$300 \mathrm{~s}$. In the case of AVR, 1.0-2.0 g of tranexamic acid was administered intravenously before the procedure. The dose of UFH was 350-400 IU/kg body weight to achieve ACT of 350-400 s. After procedures UFH action was reversed by protamine sulfate in a 0.8-1: 1 ratio.

All patients received aspirin prophylaxis. Patients on chronic aspirin prophylaxis received $75 \mathrm{mg}$ o.d. of drug until the day of the procedure. Aspirin naivve patients received a loading dose of $300 \mathrm{mg}$ of aspirin on the day preceding interventions. Starting from day of the procedures, long-term aspirin prophylaxis was recommended. After TAVI $75 \mathrm{mg}$ o.d. of aspirin, and after AVR $150 \mathrm{mg}$ o.d. of aspirin was recommended. During the first 2 post-procedural days $40 \mathrm{mg}$ o.d. of low molecular weight heparin was administered subcutaneously in all patients. In TAVI patients, clopidogrel prophylaxis with $75 \mathrm{mg}$ o.d. was started not earlier than $24 \mathrm{~h}$ after the procedure, only if proper hemostasis was achieved. In case of bleeding complications clopidogrel administration was postponed until the recovery of proper hemostasis.

\section{Hemostasis analyses}

All tests were performed at 3 time points: within $24 \mathrm{~h}$ before, directly after (30 min-1 h after protamine administration), and $24 \mathrm{~h}$ after procedures (before clopidogrel administration in TAVI patients).

Global hemostasis including intrinsic, extrinsic coagulation pathways, platelets and fibrinogen activity were assessed by thromboelastometry, with the ROTEM delta device (Tem International GmbH, Germany), using rexTEM (thromboplastin, extrinsic coagulation pathway and platelet activity), inTEM (ellagic acid, intrinsic coagulation pathway), fibTEM (cytochalasin D - platelet inhibitor, fibrinogen concentration and fibrin polymerization ability), hepTEM (heparinase - heparin inactivation, and intrinsic coagulation pathway), and starTEM tests (calcium chloride, recalcination of citrated blood). The main parameters which reflect hemostasis are: coagulation time (CT) time from the beginning of the test until the clot reaches $2 \mathrm{~mm}$ of amplitude - activation of plasma coagulation

Table I. Inclusion and exclusion criteria Inclusion criteria

- Elective, isolated TAVI or AVR with bioprosthesis implantation
- Sue to severe AS
- Written consent to participate in the study
- Age $\geq 70$ years old
Exclusion criteria
- Indication for dual antiplatelet therapy before AVR and TAVI
- Indication for oral vitamin K antagonist treatment before/after
AVR and TAVI
- Blood and blood products transfusion within 7 days before AVR
and TAVI
- Use of non-steroidal anti-inflammatory drugs (NSAIDs) or
GPIIb/IIla antagonist during $48 \mathrm{~h}$ before AVR and TAVI
- Thrombocytopenia with PLT count < $100000 / \mu l$ during $24 \mathrm{~h}$
before AVR and TAVI
- Hemoglobin < 10 g/dl during $24 \mathrm{~h}$ before AVR and TAVI
- Acute coronary syndrome with PCI or planned PCI $\leq 90$ days
before enrollment
- Coronary artery bypass grafting (CABG) $\leq 6$ months before
- enrollment
- Aortic valve intervention $\leq 6 \mathrm{months}$ before enrollment
- Recognized congenital or acquired coagulation system disor-
- Aers before enrollment
- Active infection, liver insufficiency


factors and platelets; clot formation time (CFT) - time when clot firmness is increasing from $2 \mathrm{~mm}$ to $20 \mathrm{~mm}$ - the effectiveness of primary and secondary hemostasis to form the clot; maximum clot firmness (MCF), maximum clot size - overall hemostatic potential; amplitude time $(A x)$ - clot firmness at the respective time points after CFT; maximum lysis (ML), the percentage of clot reduction in comparison to MCF - maximal lysis detected during the run time.

Platelet function was assessed using impedance aggregometry, Multiplate analyzer (Roche Diagnostics Ltd., Switzerland), and ADPtest, ASPItest, TRAPtest, RISTOtest. Platelet reactivity in ASPItest and ADPtest reflects the sensitivity to antiplatelet agents - aspirin and inhibitors of P2Y12 receptors. TRAPtest and RISTOtest expressed ability of platelets to activate in response to potent platelet activators - thrombin and VWF. Results of RISTOtest indirectly reflect the VWF activity.

The operating principles of the Multiplate and ROTEM delta analyzer have been described elsewhere [16, 17, 23, 24].

Additionally, standard blood count tests with the assessment of hemoglobin concentration - Hgb $(\mathrm{g} / \mathrm{dl})$, platelet count - PLT (1000/l), international normalized ration (INR) and activated partially thromboplastin time (APTT) were performed at the same time points.

Bleeding associated with TAVI and AVR was defined according to the Valve Academic Research Consortium 2 definition, including major and life-threatening/disabling bleeding (Table II).

\section{Ethics}

The study was performed in compliance with the Declaration of Helsinki, and was approved by the Local Ethics Committee. Written informed consent was obtained from all individuals.

\section{Statistical analysis}

Continuous variables were presented as means and standard deviations or medians and interquartile range for Gaussian and non-Gaussian distribution of the variable respectively. In statistical analyses the parametric t-test or nonparametric Mann-Whitney $U$ test was performed for normal and non-normal variables respectively. Multivariate analysis of variance (MANOVA) with post hoc analysis was performed for the multivariate analyses. The value $p<0.05$ was considered statistically significant. All statistical analyses were performed using Statistica 12.0 software (StatSoft, Inc. 2014. Statistica, version 12).

\section{Results}

We enrolled 30 patients, of whom 15 underwent AVR, and 15 were subjected to TAVI. Patients who underwent TAVI had significantly higher surgical risk, due to the greater number of comorbidities (Table II). Left ventricle ejection fraction and related with it maximal transvalvular velocity were higher in patients subjected to AVR. Otherwise, the study population was well balanced in terms of clinical characteristics.

\section{Thromboelastometry}

Patients subjected to AVR had significant hemostasis disorders related to the procedure. In comparison to initial parameters, directly after the procedure external pathway initiated hemostasis was characterized by prolonged activation of plasma factors and platelets (CT; $p=0.04$ ), prolonged clot formation (CFT; $p=0.01$ ) and reduced clot firmness at each time point (A5-A25, MCF; $p=0.03)$. Within the next $24 \mathrm{~h}$ after AVR all hemostasis parameters were significantly improved, reaching values similar to the initial ones. Comparison of rexTEM to fibTEM results proved that peri-procedural hemostasis disorders in AVR result from procedure-related fibrinogen deficiencies, with no capacity for proper clot formation (lack of CFT in fibTEM) and significant reduction in clot firmness directly after the procedure (Figures 1, 2). After $24 \mathrm{~h}$ fibrinogen concentration and fibrin polymerization abilities were restored to basic values, which allowed for formation of a clot with proper firmness (Figure 2).

Patients subjected to TAVI had similar results in external pathway initiated hemostasis (rexTEM) with significantly prolonged clot formation (CFT; $p=0.0004)$, and reduced clot firmness at each stage of test running (A5A25), including maximal clot firmness (MCF; $p=0.004$ ) directly after the procedure. Time of plasma factor activation was not disturbed (CT; $p=0.55)$. Interestingly, $24 \mathrm{~h}$ after the procedure hemostasis had not been restored completely, proving still significantly prolonged clot formation (CFT; $p=0.05$ ), and significantly reduced clot firmness (MCF; $p=0.019$ ). On the other hand, fibrinogen concentration and fibrin polymerization were not substantially disrupted directly after TAVI. Comparison of rexTEM and fibTEM results suggested that the peri-procedural hemostasis disorders in TAVI cohort resulted mainly from platelet dysfunction (Figures 1, 2).

Comparison of the results between TAVI and AVR cohorts proved a similar range of external pathway hemostasis disorders directly after the procedures (Figure 1). However, $24 \mathrm{~h}$ after interventions, hemostasis in the TAVI cohort was still impaired in contrast to almost completely recovered hemostasis after AVR (Figure 1). Comparison of fibTEM and rexTEM results between cohorts showed that hemostasis alterations in the case of AVR result from fibrinogen dysfunction, and platelet dysfunction in the case of TAVI (Figures 1, 2). No enhanced clot lysis was observed early after the procedures.

\section{Platelet reactivity}

Results of ASPItest in AVR patients proved a significant decrease in platelet reactivity directly after the pro- 
Table II. Clinical characteristics of study population and procedural outcomes

\begin{tabular}{|c|c|c|c|}
\hline Clinical characteristics & $\operatorname{AVR}(n=15)$ & $\operatorname{TAVI}(n=15)$ & $P$-value \\
\hline Age, mean \pm SD [years] & $74.93 \pm 5.78$ & $78.13 \pm 4.38$ & 0.13 \\
\hline EuroSCORE $\| \%$, mean \pm SD & $6.45 \pm 1.41$ & $11.48 \pm 4.14$ & $<0.01$ \\
\hline Sex - female, $n(\%)$ & $11(73.33)$ & $8(53.33)$ & 0.88 \\
\hline Hypertension, $n$ (\%) & $15(100)$ & $15(100)$ & - \\
\hline Diabetes mellitus, $n(\%)$ & $8(53.33)$ & $7(46.66)$ & 0.78 \\
\hline Coronary artery disease, $n(\%)$ & $8(53.33)$ & $10(66.66)$ & 0.07 \\
\hline Previous PCI, $n(\%)$ & $2(13.33)$ & $9(60)$ & 0.23 \\
\hline Previous CABG, $n(\%)$ & 0 & $3(20)$ & 0.34 \\
\hline Heart failure, $n(\%)$ & $9(60)$ & $14(93.33)$ & 0.41 \\
\hline \multicolumn{4}{|l|}{ NYHA class, $n(\%):$} \\
\hline 1 & $1(6.66)$ & 0 & \\
\hline II & $12(80)$ & $6(40)$ & 0.26 \\
\hline III & $1(6.66)$ & $9(60)$ & \\
\hline IV & $1(6.66)$ & 0 & \\
\hline Renal failure, $n(\%)$ & $4(26.66)$ & $11(73.33)$ & 0.93 \\
\hline Stroke/TIA, $n(\%)$ & $1(6.66)$ & $3(20)$ & 0.69 \\
\hline COPD, $n(\%)$ & $1(6.66)$ & $2(13.33)$ & 0.69 \\
\hline Peripheral arterial disease, $n(\%)$ & $1(6.66)$ & $7(46.66)$ & 0.28 \\
\hline Liver dysfunction, $n(\%)$ & $1(6.66)$ & $1(6.66)$ & 0.78 \\
\hline Atrial fibrillation, $n(\%)$ & $6(40)$ & $7(46.66)$ & 0.61 \\
\hline \multicolumn{4}{|c|}{ Peri-procedural parameters and in-hospital outcomes, mean \pm SD: } \\
\hline LVEF before procedure (\%) & $55.86 \pm 4.61$ & $50.6 \pm 5.27$ & 0.005 \\
\hline PGmax before procedure [mm $\mathrm{Hg}]$ & $89.46 \pm 16.76$ & $92.4 \pm 20.59$ & 0.68 \\
\hline PGmean before procedure [mm Hg] & $51.6 \pm 12.12$ & $62.0 \pm 17.08$ & 0.09 \\
\hline Vmax before procedure [m/s] & $4.8 \pm 0.5$ & $4.47 \pm 0.32$ & 0.03 \\
\hline PGmax after procedure [mm Hg] & $30.53 \pm 10.64$ & $24.33 \pm 3.28$ & 0.06 \\
\hline PGmean after procedure [mm Hg] & $15.13 \pm 7.56$ & $11.46 \pm 1.72$ & 0.09 \\
\hline Vmax after procedure $[\mathrm{m} / \mathrm{s}]$ & $2.71 \pm 0.62$ & $2.36 \pm 0.15$ & 0.13 \\
\hline Aortic regurgitation $\geq$ moderate, $n(\%)$ & 0 & 0 & - \\
\hline Anemia before procedure & $5(33.33)$ & $7(46.66)$ & 0.07 \\
\hline Hgb before procedure & $12.95 \pm 1.63$ & $12.07 \pm 1.00$ & 0.08 \\
\hline Hgb directly after procedure & $10.22 \pm 1.16$ & $10.82 \pm 1.04$ & 0.2 \\
\hline Hgb $24 \mathrm{~h}$ after procedure & $11.15 \pm 0.64$ & $10.9 \pm 1.14$ & 0.32 \\
\hline PLT before procedure & $218.33 \pm 29.9$ & $190.73 \pm 47.88$ & 0.07 \\
\hline PLT directly after procedure & $139.4 \pm 28.91$ & $141.8 \pm 40.18$ & 0.86 \\
\hline PLT $24 \mathrm{~h}$ after procedure & $163.2 \pm 17.64$ & $140.6 \pm 39.79$ & 0.08 \\
\hline APTT before procedure & $29.06 \pm 2.01$ & $32.32 \pm 4.16$ & 0.01 \\
\hline APTT $24 \mathrm{~h}$ after procedure & $41.30 \pm 6.21$ & $35.64 \pm 5.77$ & 0.03 \\
\hline INR before procedure & $0.96 \pm 0.06$ & $1.21 \pm 0.63$ & 0.15 \\
\hline INR $24 \mathrm{~h}$ after procedure & $1.17 \pm 0.65$ & $1.08 \pm 0.24$ & 0.21 \\
\hline \multicolumn{4}{|l|}{ Drainage [ml]: } \\
\hline $12 \mathrm{~h}$ after AVR & $315.33 \pm 150.04$ & - & \\
\hline In total & $447 \pm 161.29$ & & \\
\hline Reoperation due to bleeding, $n$ (\%) & $2(13.33)$ & $3(20)$ & 0.46 \\
\hline Bleeding, $n(\%)^{*}$ & $3(20)$ & $6(40)$ & 0.3 \\
\hline \multicolumn{4}{|l|}{ Transfusion, $n$ (\%): } \\
\hline PRBC & $4(26.66)$ & $7(46.66)$ & 0.88 \\
\hline FFP & $3(20)$ & $1(6.66)$ & 0.04 \\
\hline
\end{tabular}

APTT - activated partial thromboplastin time, AVR - aortic valve replacement, CABG - coronary artery bypass grafting, COPD - chronic obstructive pulmonary disease, FFP - fresh frozen plasma, Hgb - hemoglobin, INR - international normalized ratio, LVEF - left ventricle ejection fraction, PCI - percutaneous coronary intervention, PG - transvalvular pressure gradient, PLT - platelet count, PRBC - packed red blood cells, TIA - transient ischemic attack. *According VARC 2 scale. Kappetein AP, et al. Eur Heart J 2012; 33: 2403-18. 

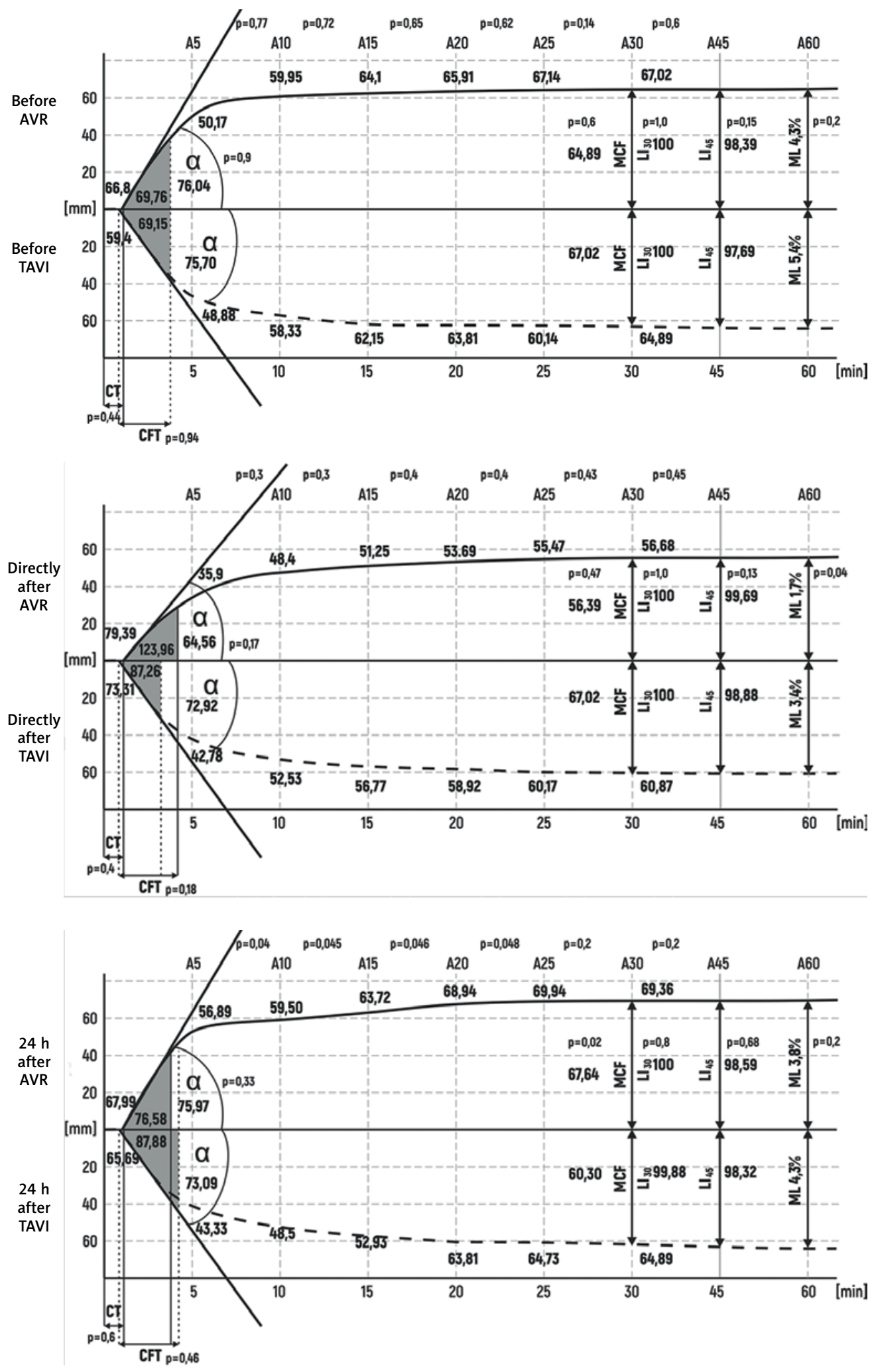

Figure 1. Changes in thromboelastometry rexTEM test related to AVR vs. TAVI. Comparison of median values of CT, CFT, A5-A30, MCF, LI, ML between cohorts 

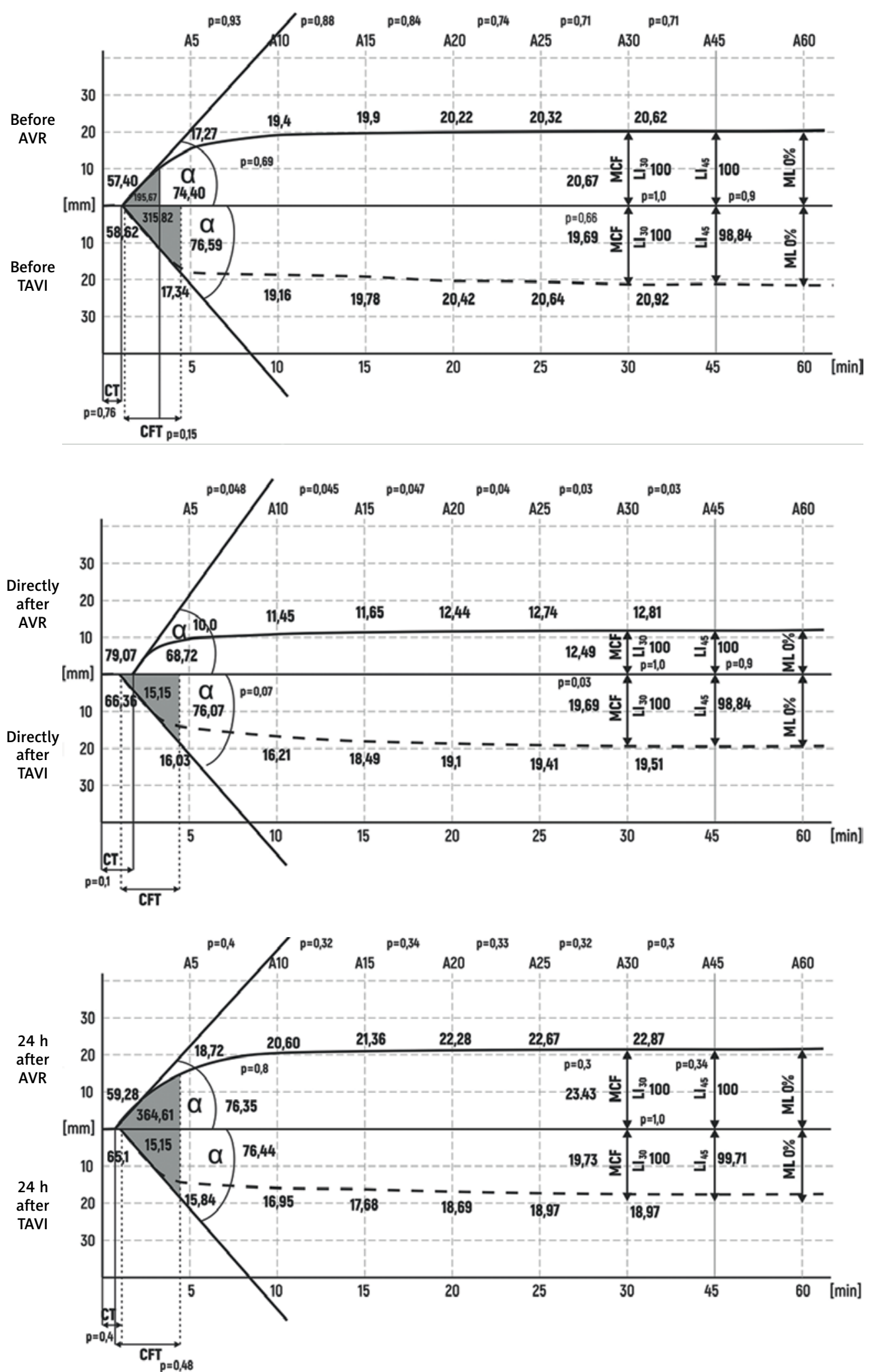

Figure 2. Changes in thromboelastometry fibTEM test related to AVR vs. TAVI. Comparison of median values of CT, CFT, A5-A30, MCF, LI, ML between cohorts 
cedure $(p<0.0001)$, which returned to basic values at $24 \mathrm{~h}$ after it $(p=\mathrm{NS})$. In the TAVI cohort platelet reactivity in ASPItest significantly decreased directly after the procedure $(p<0.0001)$, and remained significantly reduced also at $24 \mathrm{~h}$ after it, in comparison to initial values $(p<$ $0.0001)$. Comparing results of both groups, TAVI patients had significantly higher sensitivity to aspirin before and after the procedure in comparison to AVR patients, in whom quick recovery to lower sensitivity to aspirin was noted (Figure $3 \mathrm{~A}$ ).

A

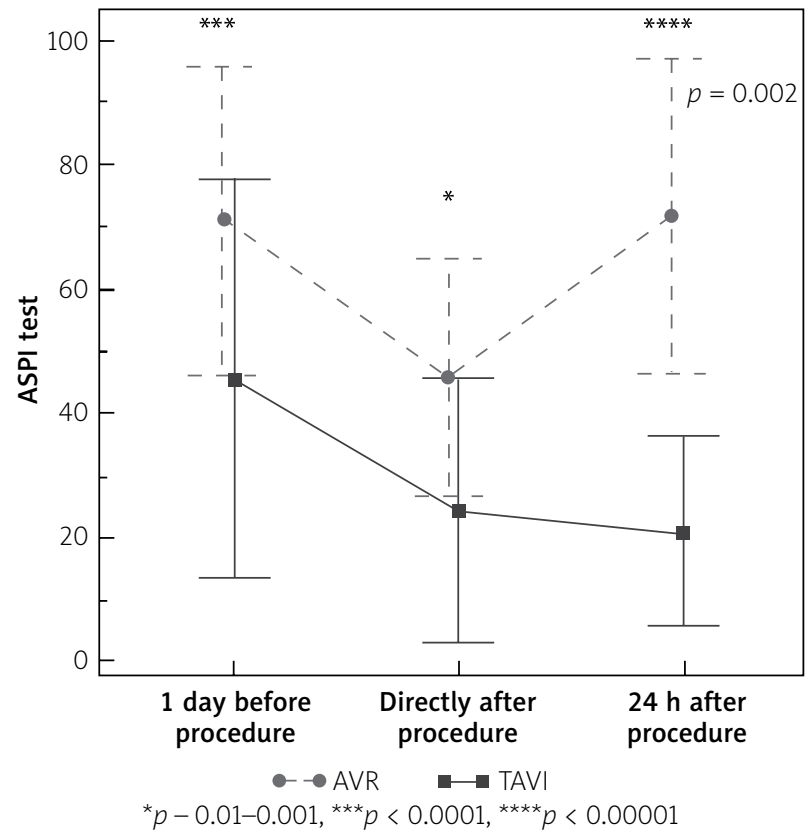

C

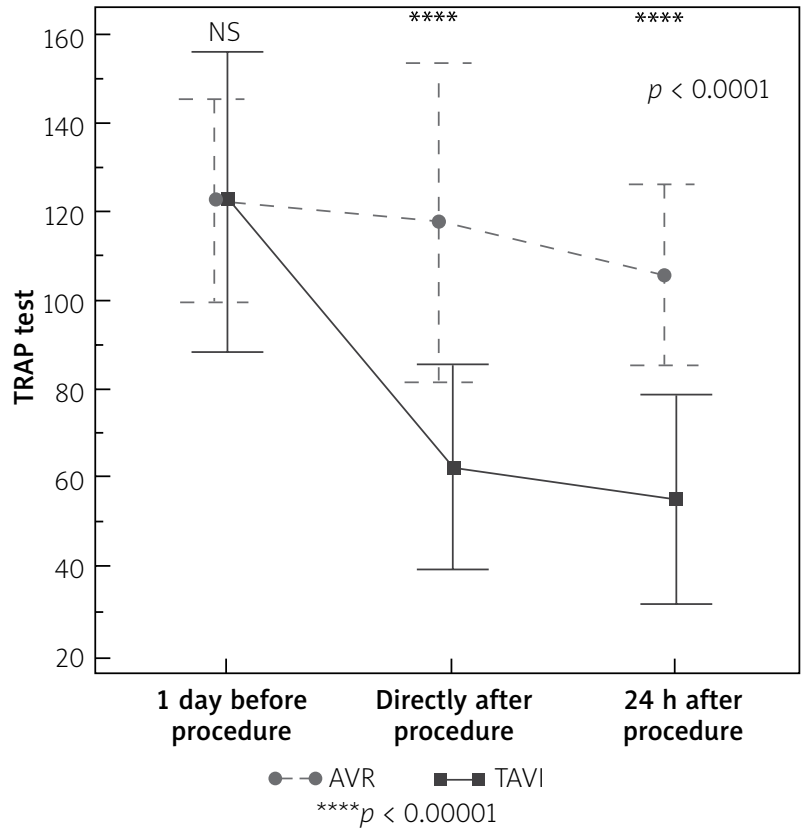

Results of ADPtest proved that only TAVI patients experienced a significant reduction in platelet reactivity directly after the procedure $(p<0.00001)$. Furthermore, this decrease persisted during the next $24 \mathrm{~h}(p<0.00001$, in comparison to initial values). In contrast, a negligible drop in platelet reactivity directly after and $24 \mathrm{~h}$ after the procedures characterized patients subjected to AVR ( $p=$ 0.1). Comparing platelet reactivity in both groups, initial sensitivity to P2Y12 inhibitors in TAVI patients was significantly greater $(p<0.01-0.001)$ and became ever more

\section{B}

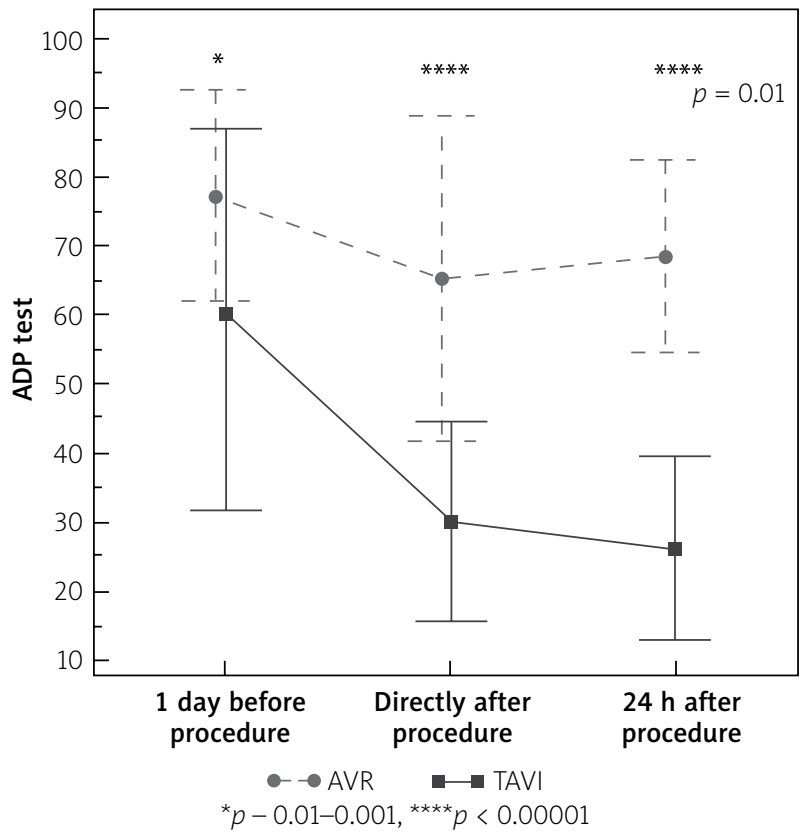

D

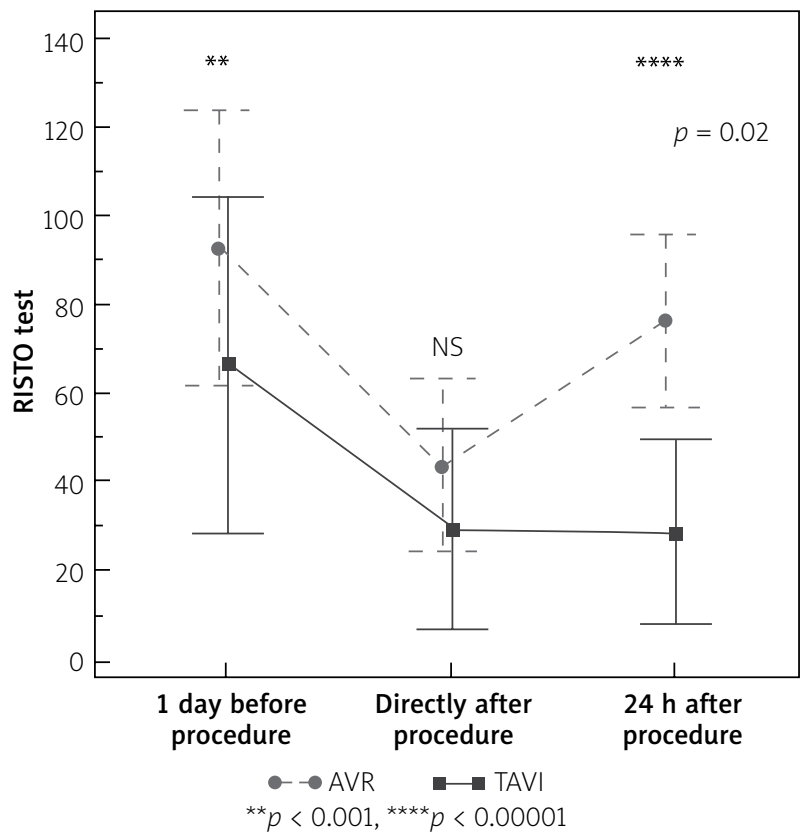

Figure 3. Comparison of changes in platelet reactivity related to AVR vs. TAVI. A - COX-1 activity, B - P2Y12 receptor activity, $\mathbf{C}$ - PAR-1 receptor activity, $\mathbf{D}-\mathrm{vWF}$ activity 
profound within $24 \mathrm{~h}$ after the procedure in comparison to AVR patients (Figure $3 \mathrm{~B}$ ).

Furthermore, results of TRAPtest proved that although initial platelet reactivity in response to thrombin receptor activation was comparable, TAVI patients experienced a significant decline in platelet reactivity directly and at $24 \mathrm{~h}$ after the procedure $(p<0.00001)$, while in patients subjected to AVR no significant changes in platelet reactivity were noted $(p>0.1$ ) (Figure $3 C$ ).

In RISTOtest AVR patients had a significant decrease in VWF activity directly after the procedure $(p<0.00001)$, with significant recovery after $24 \mathrm{~h}(p<0.00001)$. TAVI patients also experienced a significant decrease in vWF activity directly after the procedure $(p<0.0001)$. However, no recovery of VWF activity was noted at $24 \mathrm{~h}$ after TAVI $(p=N S)$ (Figure 3 D).

\section{Standard laboratory hemostasis parameters}

A significant reduction in platelet count after the procedures was observed in both cohorts. Comparing the results between groups, TAVI patients had a numerically lower platelet count before and $24 \mathrm{~h}$ after the procedure in comparison to patients subjected to AVR (Figure 4). Directly after the procedure platelet count was slightly higher in TAVI than in AVR cohort. However, none of these differences reached statistical significance. Hemoglobin concentration did not differ between groups. Activated partial thromboplastin time was significantly longer before the procedure in the TAVI cohort, while after the procedures the AVR cohort had higher APTT values (Table II).

\section{Discussion}

Due to ageing of populations and increasing prevalence of degenerative AS in highly developed countries, the number of AVR and TAVI procedures in Europe and North America is constantly increasing [7, 25].

Despite the high effectiveness, these procedures are invariably associated with increased risk of bleeding and thromboembolic events, with early bleeding as the most frequent [7-11]. In the search of causes of this phenomenon, currently attention is mainly focused on not fully evidence-based and arbitrarily recommended antithrombotic prophylaxis after these procedures [7, 26]. Peri-procedural hemostasis alterations associated with AVR and TAVI might be relevant for this phenomenon, likewise for the effectiveness and safety of peri-procedural antithrombotic prophylaxis. Yet, until now thorough analyses of hemostatic potential and platelet reactivity in the strict peri-procedural period of AVR and TAVI have never been undertaken.

It is known that patients with AS are affected by hemostasis alteration [1-6]. Initial hemostasis disorders related to AS are bidirectional, with proven high platelet activation and increased thrombin generation on one hand, and acquired deficiency of VWF or the platelet shedding phenomenon on the other [1-6, 27]. Additionally, the impact of advanced age on platelet function with increased platelet reactivity and depletion in plasma factor XIII were previously described [28-31]. Due to these alterations, AS patients are at increased risk of thromboembolic events and bleeding simultaneously [28, 31-33]. Given the fact that initially hemostasis disorders may overlap with disturbances related to the procedures themselves, the etiology of peri-procedural hemostasis disorders in AS patients seems to be multifactorial, complex, and not fully investigated.

Hemostasis disorders associated with AVR are partially explained by the use of extracorporeal circulation. The insufficient hemocompatibility of cardiopulmonary bypass (CPB) devices, which are negatively charged and not covered with endothelium, causes activation of plasma coagulation factors, platelets, complement components, endothelial cells and leucocytes [8, 16, 18, 27]. Subsequently, fibrinogen and VWF are adsorbed onto the surface of CPB, providing a nidus for platelet adhesion and aggregation. This results in constant activation and consumption of plasma coagulation system components expressed in hypofibrinogenemia, high platelet thrombogenicity, and a global inflammatory response [34, 35]. Non-physiological turbulent blood flow with high shear forces and areas of stasis in CPB potentiate these alterations [34, 35]. On top of this, dilutional coagulopathy and prolonged activation of coagulation factors caused by hypothermia have been proven [36]. Despite the substantial hemostasis disorders associated with AVR, quick restoration of vWF multimers and fibrinogen within $24 \mathrm{~h}$ after this procedure was reported [22]. Similarly, increased platelet reactivity with increased thrombogenicity within 3 months after AVR has been proven $[3,20,21]$.

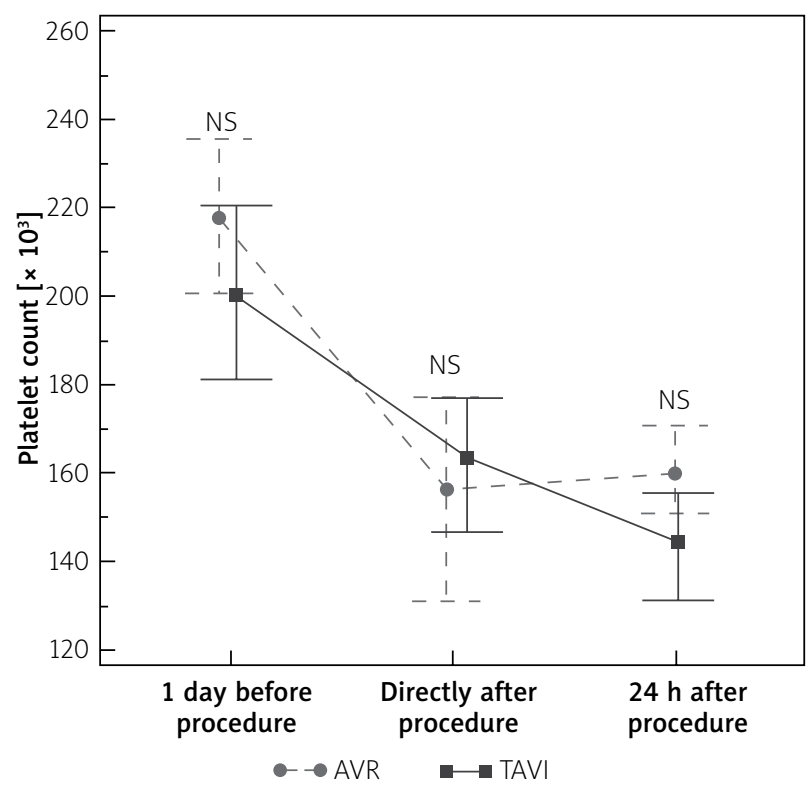

Figure 4. Comparison of changes in platelet count related to AVR vs. TAVI 
Data on hemostasis alterations associated with TAVI have considered only relevant thrombocytopenia, increased platelet activation, expressed in release of microparticles, and immediate restoration of VWF multimers after the procedure [12-15, 33, 37, 38].

The importance of our study is expressed in a few novel results.

We performed detailed analysis of platelet reactivity disturbances related to AVR and TAVI. Furthermore, we compared the two procedures, proving that the peri-procedural hemostatic profile of patients subjected to AVR differs in comparison to that of those subjected to TAVI.

In the case of platelet reactivity we found that patients subjected to AVR had only a transient increase in sensitivity to aspirin with quick recovery to baseline reactivity. Of note, this finding might not express the quick restoration of young, reactive platelets, but rather the result of constant activation related to CPB and heparin, as was suggested previously [30, 34, 35].

Furthermore, AVR patients presented significant, but only procedure-related depletion in VWF activity, with restoration within $24 \mathrm{~h}$ after AVR. This finding is contradictory to previous reports, which showed that reduced VWF activity concerns AS patients before AVR, with complete restoration within the next $24 \mathrm{~h}$ [34].

In the case of TAVI, we proved that the main cause of peri-procedural hemostasis disorders results from platelet dysfunction. We found significant, procedure-related impairment of platelet reactivity, which in contrast to AVR did not improve after $24 \mathrm{~h}$.

Interestingly, platelet sensitivity to aspirin before and after TAVI was higher in comparison to AVR, although both cohorts received equal doses of aspirin before the procedures, and AVR patients received a double dose of aspirin after surgery. Similarly, initial sensitivity to P2Y12 inhibitors was significantly higher in the TAVI cohort and substantially increased after TAVI. This change was significantly stronger than after AVR, though none of the TAVI patients received clopidogrel before and within $24 \mathrm{~h}$ after the procedures. Furthermore, in contrast to the previous reports $v W F$ activity after TAVI did not recover within $24 \mathrm{~h}$, though no significant aortic regurgitation was found [13, 33].

It is difficult to compare our outcomes to studies which adopted different time points and parameters for hemostasis assessment after TAVI, especially when their results are divergent [12-15, 33, 37, 38].

Jung et al. observed that, 1 week after TAVI, serum concentration of platelet microparticles, a marker of platelet activation, was lower than before the procedure, which significantly increased afterwards [12]. Another, small sample size study investigated viscoelastic properties of clot formation early after TAVI [14]. So far, only one study has compared platelet function and global hemostasis in patients subjected to AVR and TAVI [15]
Similarly to our observations, the researchers observed a drop with quick restoration in platelet reactivity after $A V R$, and significantly impaired platelet function directly after TAVI [15], which did not recover to initial values until the last assessment. In contrast, fibrinogen function was only mildly impaired after AVR with rapid reconstitution to values which even exceeded the initial ones, and a significant procedure-related drop in platelet count occurred only in the AVR cohort [15].

We are aware that the results of platelet reactivity analyses may be altered by platelet count. Similarly as previous studies, we found that platelet count decreased significantly after both interventions [37-39]. Yet, the observed differences in platelet reactivity between AVR and TAVI cannot be simply explained by procedure-related thrombocytopenia, since both study cohorts had a similar platelet count at each time point of assessment.

A potential explanation of this phenomenon might be platelet receptor shedding related to the TAVI procedure $[27,35]$. However, if so, the AVR procedure and shear forces of CPB should be more traumatic for platelets. Another cause of this finding might be diminished platelet regeneration after TAVI. It was proved that elderly people have reduced potential to regenerate blood components after bleeding, which was expressed in a reduced number of reticulated, more reactive platelets [40]. However, AVR is associated with greater blood loss than TAVI; therefore, impairment in platelet reactivity should be more profound in AVR patients.

\section{Study limitations}

Our study has an explorative and hypothesis-generating nature; therefore, the results should be perceived as preliminary. The explorative character determines the small sample size of the study, which together with limited diagnostic tools and time points of assessment do not allow us to draw too far-reaching conclusions. The small sample size was responsible for the high rate of noted bleeding. It also made it impossible to correlate discovered hemostasis disorders with hemorrhagic events. Furthermore, differences in procedures limit the value of comparative analysis of hemostasis disorders between the interventions. The two procedures also differ in terms of doses of UFH and expected level of ACT during the intervention. Thus, we did not analyze the results of inTEM tests, which express the impact of UFH and protamine on hemostasis. Durative hemostasis disorders may be responsible for early bleeding related to aortic valve interventions. Our study may become a basis for further thorough analyses, designed to verify this hypothesis.

\section{Conclusions}

Surgical and transcatheter and aortic valve procedures are associated with substantial and diverse 
peri-procedural hemostasis disorders. Surgical aortic valve replacement is characterized by hypofibrinogenemia and vWF depletion, which quickly recover within $24 \mathrm{~h}$ after the procedure. Transcatheter aortic valve implantation is characterized by early significant impairment of platelet function with increased sensitivity to antiplatelet agents, which is not recovered at $24 \mathrm{~h}$ after the procedure. Therefore early dual antiplatelet prophylaxis after TAVI requires careful consideration.

\section{Conflict of interest}

The authors declare no conflict of interest.

\section{References}

1. Le Tourneau T, Breyne J, Susen S. Ambivalent effect of aortic stenosis on von Willebrand factor and thrombin generation. Is transvalvular gradient the guilty party? Heart 2011; 97:1997-8.

2. Natorska J, Bykowska K, Hlawaty M, et al. Increased thrombin generation and platelet activation are associated with deficiency in high molecular weight multimers of von Willebrand factor in patients with moderate-to-severe aortic stenosis. Heart 2011; 97: 2023-8.

3. Badar ER, Gremmel T, Schneller A, et al. High levels of plateletmonocyte aggregates after valve replacement for aortic stenosis: relation to soluble P-selectin and P-selectin glycoprotein ligand-1 genes. Thrombosis Res 2012; 129: 453-8.

4. Aslan JE, Itakura A, Haley KM, et al. p21 activated kinase signaling coordinates glycoprotein receptor VI-mediated platelet aggregation, lamellipodia formation, and aggregate stability under shear. Arterio Thromb Vasc Biol 2013; 33: 1544-51.

5. Chirkov YY, Holmes AS, Willoughby SR, et al. Association of aortic stenosis with platelet hyperaggregability and impaired responsiveness to nitric oxide. Am J Cardiol 2002; 90: 551-4.

6. Diehl P, Nagy F, Sossong V, et al. Increased levels of circulating microparticles in patients with severe aortic valve stenosis. Thromb Haemost 2008; 99: 711-9.

7. Baumgartner H, Falk V, Bax J, et al. 2017 ESC/EACTS Guidelines for the management of valvular heart disease. Eur Heart J 2017; 38: 2739-91.

8. Kulik A, Rubens FD, Wells PS, et al. Early postoperative anticoagulation after mechanical valve replacement: a systematic review. Ann Thorac Surg 2006; 81: 770-81.

9. Czerwińska-Jelonkiewicz K, Zembala M, Dąbrowski M, et al. Can TAVI patients receive aspirin monotherapy as patients after surgical aortic bioprosthesis implantation? Data from the Polish Registry-POLTAVI. Int J Cardiol 2017; 227: 305-11.

10. Ando T, Akintoye E, Holmes AA, et al. Clinical end points of transcatheter aortic valve implantation compared with surgical aortic valve replacement in patients $<65$ years of age (from the National Inpatient Sample Database). Am J Cardiol 2018; 122: 279-83.

11. Schymik G, Varsami C, Bramlage P, et al. Two-year outcomes of transcatheter compared with surgical aortic valve replacement in "minimal-risk" patients lacking EuroSCORE co-morbidities (from the TAVIK Registry). Am J Cardiol 2018; 122: 149-55.

12. Jung $C$, Lichtenauer $M$, Figulla $H R$, et al. Microparticles in patients undergoing transcatheter aortic valve implantation (TAVI). Heart Vessels 2017; 32: 458-66.
13. VanBelle E, Rauch A, Vincent F, et al. Von Willebrand factor multimers during transcatheter aortic-valve replacement. N Engl J Med 2016; 375: 335-44.

14. Rymuz B, Zbroński K, Ścisło P, et al. Thromboelastography for predicting bleeding in patients with aortic stenosis treated with transcatheter aortic valve implantation. Kardiol Pol 2018; 76: 418-25.

15. Uhle F, Castrup C, Necaev AM, et al. Inflammation and its consequences after surgical versus transcatheter aortic valve replacement. Artificial Organs 2018; 42: E1-12.

16. Kozek-Langenecker SA, Ahmed AB, Afshari A, et al. Management of severe perioperative bleeding. Guidelines from the European Society of Anaesthesiology. First update 2016. Eur J Anaesthesiol 2017; 34: 332-95.

17. Gorlinger K, Dirkmann D, Hanke AA, et al. First-line therapy with coagulation factor concentrates combined with point-of-care coagulation testing is associated with decreased allogeneic blood transfusion in cardiovascular surgery: a retrospective, single-center cohort study. Anesthesiology 2011; 115: 1179-91.

18. Hartmann M, Sucker C, Boehm O, et al. Effects of cardiac surgery on hemostasis. Transfus Med Rev 2006; 20: 230-41.

19. Mahla E, Suarez TA, Bliden KP, et al. Platelet function measurement based strategy to reduce bleeding and waiting time in clopidogrel treated patients undergoing coronary artery bypass graft surgery: the timing based on platelet function strategy to reduce clopidogrel associated bleeding related to CABG (TARGETCABG) study. Circ Cardiovasc Interv 2012; 5: 261-9.

20. Conrad J, Horellou MH, Baillet M, et al. Plasma beta-thromboglobulin in patients with valvular heart disease with or without valve replacement: relationship with thromboembolic accidents. Eur Heart J 1984; 5 Suppl D: 13-8.

21. Le Guyader A, Watanabe R, Berbe J, et al. Platelet activation after aortic prosthetic valve surgery. Interactive Cardio-Vasc Thoracic Surg 2006; 5: 60-4.

22. Goldsmith IR, Blann AD, Patel RY, Lip GY. Plasma fibrinogen, soluble P-selectin, and von Willebrand factor in aortic valve disease: evidence for abnormal haemorheology, platelet activation, and endothelial dysfunction. Heart 2000; 83: 577-8.

23. Weber $\mathrm{CF}$, Gorlinger $\mathrm{K}$, Meininger D, et al. Point-of-care testing: a prospective, randomized clinical trial of efficacy in coagulopathic cardiac surgery patients. Anesthesiology 2012; 117: 531-47.

24. Aradi D, Storey RF, Komocsi A, et al. Expert position paper on the role of platelet function testing in patients undergoing percutaneous coronary intervention. Eur Heart J 2014; 35: 209-15.

25. Nkomo VT, Gardin JM, Skelton TN, et al. Burden of valvular heart diseases: a population-based study. Lancet 2006; 368: 1005-11.

26. Lip GY, Windecker S, Huber K, et al. Management of antithrombotic therapy in atrial fibrillation patients presenting with acute coronary syndrome and/or undergoing percutaneous coronary or valve interventions. Eur Heart J 2014; 35: 3155-79.

27. Casan J, Andrews RK, Gardiner EE, et al. Mechanisms of platelet dysfunction in patients with implantable devices. Semin Thromb Hemost 2018; 44: 12-9.

28. Andreott F, Rocca B, Husted S, et al. Antithrombotic therapy in the elderly: expert position paper of the European Society of Cardiology Working Group on Thrombosis. Eur Heart J 2015; 36: 3238-49.

29. Breet NJ, van Donkersgoed HE, van Werkum JW, et al. Is platelet inhibition due to thienopyridines increased in elderly patients, in patients with previous stroke and patients with low body 
weight as a possible explanation of an increased bleeding risk? Neth Heart J 2011; 19: 279-84.

30. Cuisset T, Quilici J, Grosdidier C, et al. Comparison of platelet reactivity and clopidogrel response in patients $<75$ years versus $>75$ years undergoing percutaneous coronary intervention for non-ST-segment elevation acute coronary syndrome. Am J Cardiol 2011; 108: 1411-6.

31. Silvain J, Cayla G, Hulot JS, et al. High on-thienopyridine platelet reactivity in elderly coronary patients: the SENIOR-PLATELET study. Eur Heart J 2012; 33: 1241-50.

32. Pate GE, Chandavimol M, Naiman SC, Webb JG. Heyde's syndrome: a review. J Heart Valve Dis 2004; 13: 701-12.

33. Godino C, Lauretta L, Pavon AG, et al. Heyde's syndrome incidence and outcome in patients undergoing transcatheter aortic valve implantation. J Am Coll Cardiol 2013; 61: 687-9.

34. Heilmann C, Geisen U, Beyersdorf F, et al. Acquired von Willebrand syndrome in patients with extracorporeal life support (ECLS). Intensive Care Med 2012; 38: 62-8.

35. Lukito P, Wong A, Jing J, et al. Mechanical circulatory support is associated with loss of platelet receptors glycoprotein Ibalpha and glycoprotein VI. J Thromb Haemost 2016; 14: 2253-60.

36. Keenan JE, Wang H, Gulack BC, et al. Does moderate hypothermia really carry less bleeding risk than deep hypothermia for circulatory arrest? A propensity-matched comparison in hemiarch replacement. J Thorac Cardiovasc Surg 2016; 152: 1559-69.

37. Gallet R, Seemann A, Yamamoto M, et al. Effect of transcatheter (via femoral artery) aortic valve implantation on the platelet count and its consequences. Am J Cardiol 2013; 111: 1619-24.

38. Gul M, Uyarel H, Akgul O, et al. Hematologic and clinical parameters after transcatheter aortic valve implantation (TAVI) in patients with severe aortic stenosis. Clin Appl Thromb Hemost 2014; 20: 304-10.

39. McCabe JM, Huang PH, Riedl LA, et al. Incidence and implications of idiopathic thrombocytopenia follow ing transcatheter aortic valve replacement with the Edwards Sapien valves: a single center experience. Catheter Cardiovasc Interv 2014; 83: 633-41.

40. Hoffmann JJ. Reticulated platelets: analytical aspects and clinical utility. Clin Chem Lab Med 2014; 52: 1107-17. 